\title{
Pattern formation, stability and collapse in 2D driven particle systems
}

\author{
Maria R. D'Orsogna ${ }^{1}$, Yao-li Chuang ${ }^{2}$, Andrea L. Bertozzi ${ }^{1}$, Lincoln S. \\ Chayes. $^{1}$ \\ 1 Department of Mathematics, UCLA, Los Angeles, CA 90095 \\ dorsogna@math.ucla.edu \\ 2 Department of Physics, Duke University, Durham, NC 27708
}

Interacting, multi-robot systems show increasing promise for advances in exploration and defense applications. Here, we model a non-linear system of self-propelled individuals interacting via a pairwise attractive and repulsive potential. Depending on the interaction parameters, the agents may disperse, accumulate into self-organizing structures such as flocks and vortices, or collapse onto themselves. Borrowing tools from Statistical Mechanics, we discuss the connections between the $\mathrm{H}$-stable nature of the interaction potential and resulting aggregating patterns and asymptotic behaviors.

\section{Introduction}

Designing and controlling robot assemblies to achieve specific collective goals has drawn considerable interest in recent years $[1,2,3,4]$. An individual agent may be programmed to be fully autonomous and independent, but because of physical and resource constraints, its abilities may be limited. On the other hand, groups of individuals exchanging information and optimally selforganizing may have a much broader range of capabilities. Natural examples of interacting 'swarms' abound: fish, birds, bacteria and insects communicate to create complex patters with new and useful group properties $[5,6]$. These structures often form without the aid of a leader or of mediating chemical fields and only in response to local interactions. The underlying idea of robot swarming is to coordinate agent motion in a similar, intelligent manner, sometimes looking at nature for inspiration [7]. Teams of interacting artificial agents may someday be routinely used in underwater or space exploration missions, or for the completion of military and other dangerous tasks, such as land-mine detection and removal or earthquake recovery [1].

A major issue is the control of swarm size and stability with respect to constituent number. Given $N$ agents that interact through simple rules, how does the size of the swarm depend on $N$ ? Can we design interactions so that swarms 


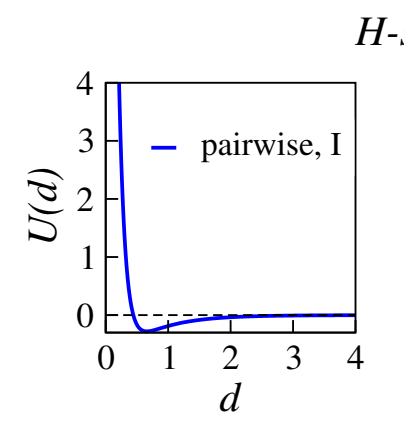

H-stable
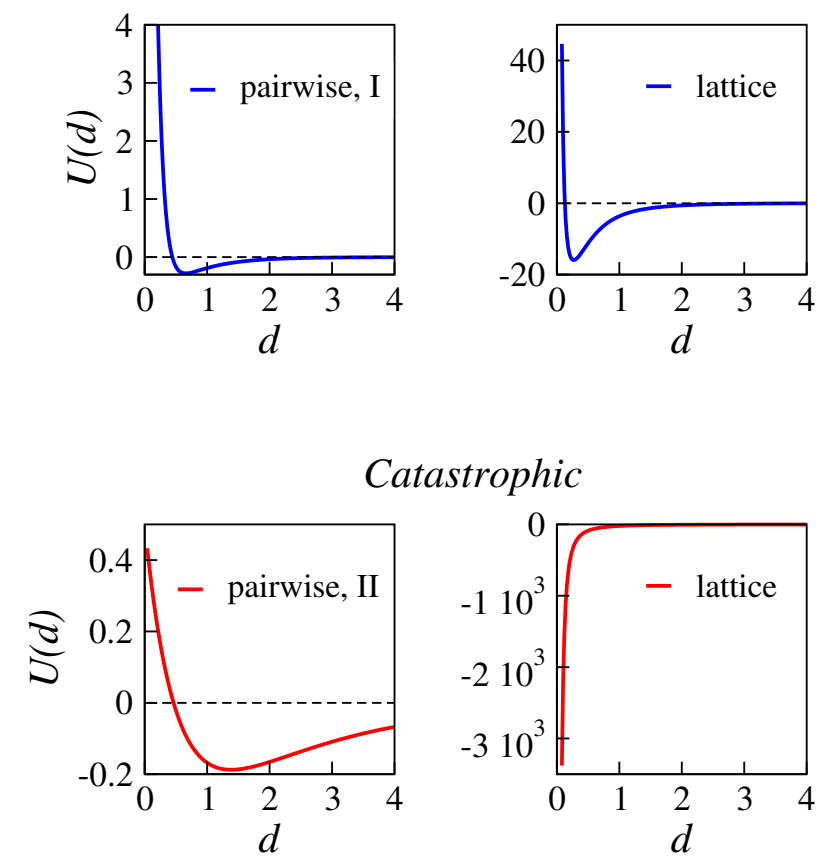

\section{Catastrophic}

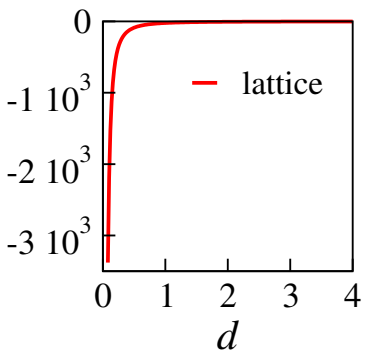

Fig. 1. H-stable and catastrophic behaviors. The pairwise panels I, II show the two-body interaction between agents. In both cases a minimum separation distance exists. If agents are placed on an infinite triangular lattice of spacing $d$, the overall energy is minimized in dramatically different ways. For the pairwise I case, in the large number limit, agents are separated by a finite distance. For the pairwise II case, the infinite system energy is minimized with lattice constant $d \rightarrow 0$. This is the catastrophic case where agents collapse upon themselves.

are stable as the number of constituents increases so that inter-agent spacings are fixed without agents collapsing or dispersing as $N$ grows? Obviously the answer depends on the particular agent-agent interaction. Here, we discuss stability and pattern formation of a prototypical system of $N$ self-propelled individuals interacting through pairwise attractive and repulsive potentials. We have addressed most of these issues in Ref. [8].

\section{Stability and Collapse}

Many-body, interacting individuals are often encountered in physical systems at the microscopic scale. Statistical mechanics aims at describing such aggregates in a more macroscopic, 'thermodynamic' way [9]. To make the passage to the macroscopic world meaningful, so that thermodynamics can be fully 


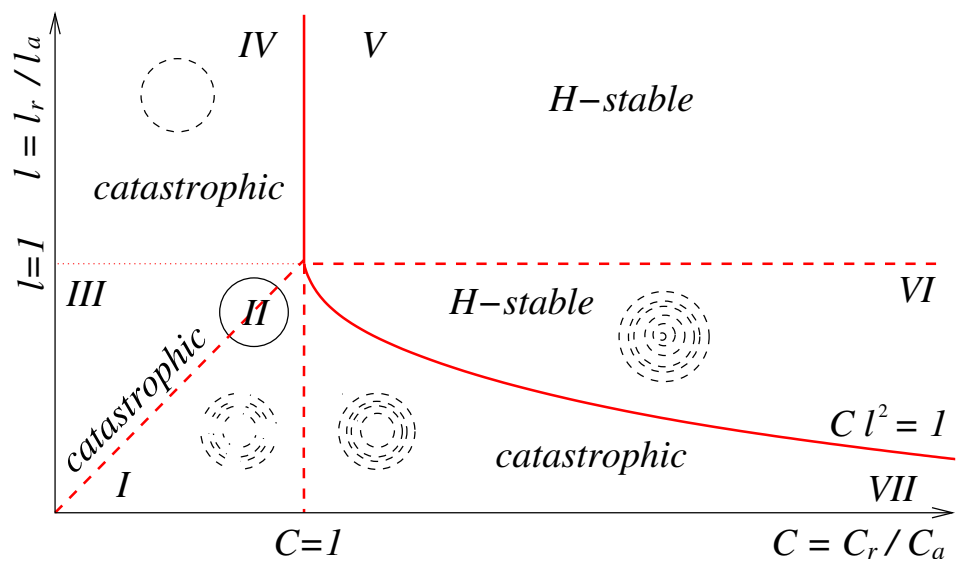

Fig. 2. H-stability phase diagram of the Morse potential (taken from Ref. [8]). Catastrophic and stable behavior are predicted as a function of the parameter ratios $\ell=\ell_{r} / \ell_{a}$ and $C=C_{r} / C_{a}$. Extrema of the potential $d_{\min }$ exist only for $\ell>\max \{1, C\}$ and for $\ell<\min \{1, C\}$. In these cases $d_{\min }=\ell_{r} \log (\ell / C) /(\ell-1)$. For each region a qualitative pattern outcome is shown. In region $\mathrm{V}$ agents disperse to infinity.

obtained, the pairwise interactions must obey specific requirements. In particular, for any arbitrarily large number $N$ of agents, if a constant $B \geq 0$ exists such that $\sum_{i} U\left(\mathbf{x}_{i}\right) \geq-N B$ the microscopic agents will not collapse onto themselves and a typical distance between individuals will be well defined. This is the fundamental property of H-stability. Systems that do not obey this constraint are called catastrophic. Upon increasing the number of agents, the latter will tend to accumulate at the same region in space. For catastrophic systems the thermodynamic limit cannot be defined. We will apply these concepts to our multi-vehicular ensembles.

Mathematically, H-stability translates to many conditions on the pairwise potential $[10,11]$. For example if its spatial integral is negative, the system is proven to be catastrophic and collapse will occur. An illustration is given in Fig. 1, where two cases of pairwise potentials and their corresponding manybody energy on an infinite lattice are shown. The two pairwise curves are qualitatively similar: both are soft-core, have a minimum and decay to zero exponentially. The pairwise potential on the right, however, subtends a negative area, signaling catastrophic behavior. This is seen, for example, as agents are placed on an infinite triangular lattice of variable lattice constant $d$. For the $\mathrm{H}$-stable potential, depicted on the left, a typical spacing emerges that minimizes the energy: agents assemble at this finite distance even as $N \rightarrow \infty$. In particular, the system behaves extensively and agents do not collapse onto each other. The right panels correspond to the catastrophic regime. Here, a minimum exists for the two body potential, as seen in the upper figure; 
however, as $N \rightarrow \infty$ global energy minimization occurs for inter-agent spacing $d=0$. The system will now collapse onto itself in the large agent limit. Similar trends persist when agents assemble on a square lattice. Other rules for H-stability are given in D. Ruelle's book [10]. Lennard-Jones and hardcore potentials are always stable: for systems interacting according to either, catastrophic behavior never occurs.

One other requisite for thermodynamic, macroscopic behavior, is that of temperedness, namely, that the long range attractive part of the potential should not be too strong. In particular, it can be shown that in $d$ dimensions the attractive part of the potential should go to zero faster than $r^{-d}$. Three dimensional gravitational interactions scale as $r^{-1}$ : the universe does not obey the laws of thermodynamics! The same can be said about Coulomb forces between same charge particles $[10,11]$. The generalized Morse potential of Eqn. 3 decays exponentially, and satisfies the temperedness condition regardless of the potential parameters. Macroscopic, extensive behavior therefore can be violated only in the catastrophic regime.

\section{The model}

We let the $1 \leq i \leq N$ discrete swarming agents be governed by the following equations of motion $[12,8]$ :

$$
\begin{aligned}
\frac{d \mathbf{x}_{i}}{d t} & =\mathbf{v}_{i}, \\
m_{i} \frac{d \mathbf{v}_{i}}{d t} & =\left(\alpha-\beta\left|\mathbf{v}_{i}\right|^{2}\right) \mathbf{v}_{i}-\nabla_{i} U\left(\mathbf{x}_{i}\right) .
\end{aligned}
$$

The self-accelerating force $\alpha \mathbf{v}_{i}$ tends to balance the self-decelerating term $\beta\left|\mathbf{v}_{i}\right|^{2} \mathbf{v}_{i}$, giving individuals the tendency to travel close to the self-propelling speed $\left|\mathbf{v}_{i}\right|=\sqrt{\alpha / \beta}$, a mechanism first introduced by Rayleigh [13, 14]. Note that the self-propelling mechanism tends to fix the magnitude of the velocity but not the direction of motion. Two major dynamical outcomes, mostly dependent on the initial conditions, are then possible: circular motion or a coherent agent drift. Interactions follow the generalized Morse potential:

$$
U\left(\mathbf{x}_{i}\right)=\sum_{j \neq i}\left[C_{r} e^{-\left|\mathbf{x}_{i}-\mathbf{x}_{j}\right| / \ell_{r}}-C_{a} e^{-\left|\mathbf{x}_{i}-\mathbf{x}_{j}\right| / \ell_{a}}\right],
$$

where $\ell_{a}, \ell_{r}$ represent the range of the attractive and repulsive part of the potential and $C_{a}, C_{r}$ are their respective amplitudes. As we shall see, the existence of a preferred speed coupled with pairwise interactions among agents leads to interesting aggregation patterns. The potential of Eqn. 3 is used as an example of soft-core potential; combinations of other attractive and repulsive 


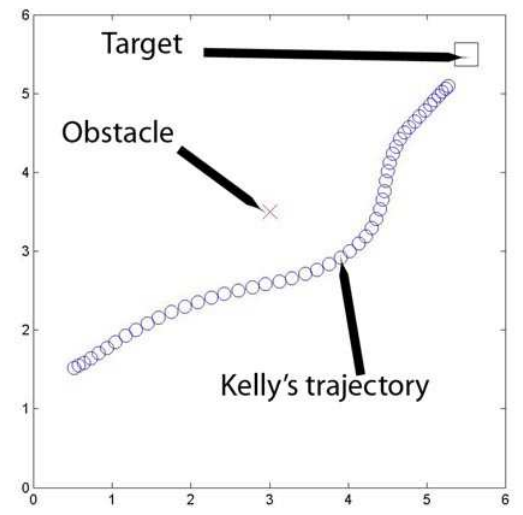

Fig. 3. A 'Kelly' vehicle guided by two virtual potentials of the Morse type: one to guide the vehicle towards a target site, the other to avoid an obstacle. From Ref. [15].

terms lead to collective trends that can be easily understood through this specific example. For simplicity, we shall only consider a 2D description and focus here on identical mass agents: $m_{i}=m$.

Actual realizations of self-propelled vehicles interacting according to virtual Morse potentials have been already introduced in the robotics literature $[1,15]$. For example, in Ref. [15], 'Kelly' vehicles were built to be self-propelled by a set of two fans separated by a variable distance and driven by virtual attractive and repulsive forces. The equations of motion for speed and angular velocity of the Kellys were directly mapped onto equations similar to those presented in Eqns. 1-3. In particular, changing the self-propulsion and the Morse parameters enabled the vehicle to effectively orient itself towards an attractive target, or to avoid multiple stationary obstacles. Further cooperative strategies could also be implemented so that a many-vehicle system could be used to effectively search more than one location. An example of the Kelly motion is given in Fig. 3.

In the remainder of this paper we discuss the consequences of decentralized control of the form presented in Eqns. 1-3 when applied to a large system of vehicles interacting with each other.

\section{Pattern formation}

In this section we apply the criteria for H-stability to the Morse potential of Eqn. 3. The choice of the parameters $C \equiv C_{r} / C_{a}$ and $\ell \equiv \ell_{r} / \ell_{a}$ determines stable or catastrophic behavior [8], as seen in the phase diagram of Fig. 2. A similar analysis can be extended to most potentials introduced in the robotics literature, for example that of Refs. [16, 17], where catastrophic behavior is seen. 


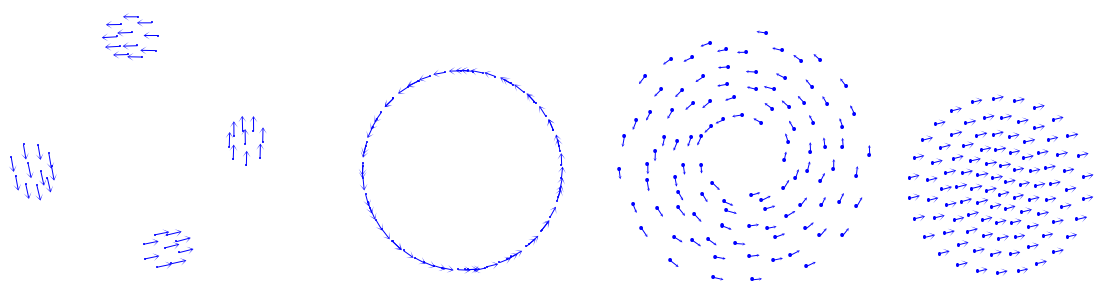

Fig. 4. Aggregating geometries for different Morse potential parameters and for $N=100$ agents. From left to right: clumps (region I), a ring (region I), a vortex and a flock (region VII).

By numerically integrating Eqns. 1-3 [18] with free boundaries and random initial conditions, we can distinguish different aggregation regimes in the $\{C, \ell\}$ phase space. All are consistent with the stable or catastrophic predictions of Fig. 2. Regions I through IV of the phase diagram define catastrophic potentials and structures decrease in size as $N$ increases. In Fig. 4 we show different aggregating patterns. Clumps form whenever the pairwise interaction admits a minimum (region I) and rings occur when that minimum is zero (region II). In regions III and IV, where the pairwise potential does not allow for a minimum, clumped rings form (not shown) as a way to minimize the total energy while keeping a constant speed. Region $\mathrm{V}$ corresponds to dispersive behavior, where the agents occupy the entire volume.

Regions VI and VII are the most interesting of the phase diagram. In the stable region VI, coherent structures can form only at relatively low values of $\alpha / \beta$, when the kinetic energy of the agents is comparable to the confining interaction potential. Swarming individuals assemble in a flock or in a disk, depending on the initial conditions; generally spacings are well defined, the motion is rigid-body like and the structures are extensive. For the case of rigid-body motion, the agents do not define a stationary center of mass, but rather the latter executes a non trivial trajectory. For larger values of $\alpha / \beta$, individuals disperse.

In the catastrophic region VII, in addition to the two extreme behaviors seen in region VI - rigid-like motion and dispersion at low and high values of $\alpha / \beta$, respectively - rotating vortices may be generated for intermediate values of $\alpha / \beta$. Here, the swarming individuals travel close to the characteristic speed $\left|\mathbf{v}_{i}\right|^{2} \sim \alpha / \beta$ and for random initial directions of motion, the center of mass is fixed. As shown pictorially in the left hand panel of Fig. 5 vortex size decreases dramatically with agent number. In the right hand side of Fig. 5 we also plot vortex area scaling with number of constituents $N$, for various $\alpha$ at fixed $\beta$ in the catastrophic regime. Note that as $N$ increases the area dramatically decreases. 

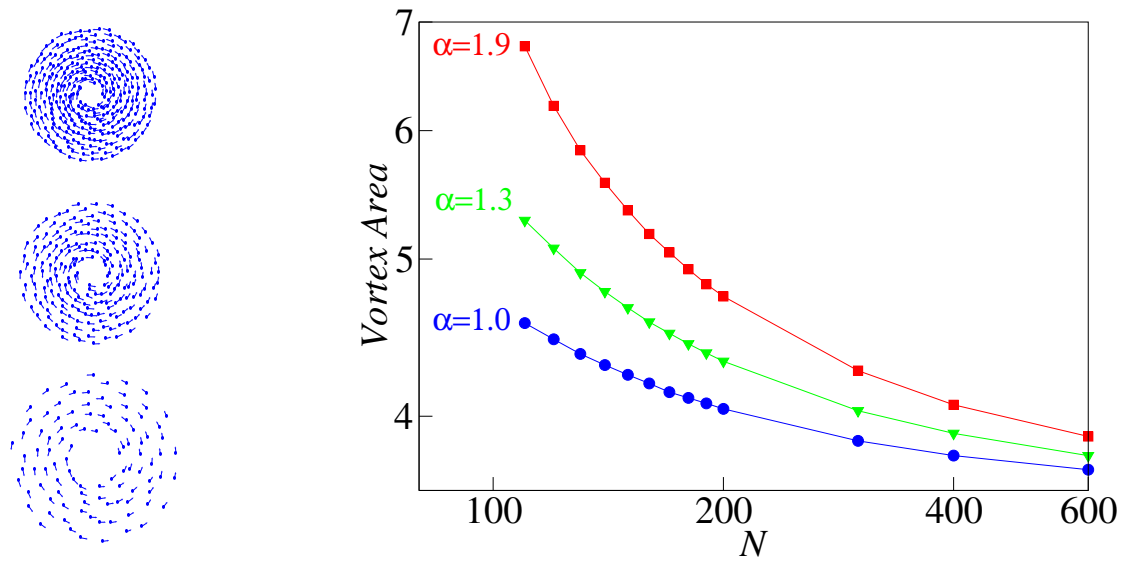

Fig. 5. Left: Swarms in the catastrophic region VII of Fig. 1. From bottom to top $N=100,200,300$. Due to the catastrophic nature of the potential, the vortex area decreases dramatically with $N$, and the density increases. The specific potential parameters are chosen as: $C_{a}=0.5, C_{r}=1, \ell_{a}=2, \ell_{r}=0.5$. The self-propelling values $\alpha$ and $\beta$ are set as: $\alpha=1.6$ and $\beta=0.5$. Right: Vortex area as a function of $N$ for various $\alpha$ in log-log scale for the same values of potential parameters. Note the collapsing trend as $N \rightarrow \infty$.

In the case of vortex motion, the dynamics of the center of mass, moving with velocity $\mathbf{V}$, can be obtained by summing Eqn. 2 over all particles $i$ with $\left|v_{i}\right|^{2}=\alpha / \beta$. We obtain:

$$
m \sum_{i} \dot{\mathbf{v}}_{\mathbf{i}} \equiv N m \dot{\mathbf{V}}=\sum_{i} \nabla_{i} U\left(\mathbf{x}_{i}\right)=0
$$

where the latter equality arises from the distance dependence of the potential in Eq. 3 and the double sum in all pairs $\{i, j\}$. Vortices thus are localized in space, to the contrary of rigid body structures for which the equality $\left|v_{i}\right|^{2}=$ $\alpha / \beta$ does not hold.

Structures such as clumps, vortices and rings generally rotate counterclockwise or clockwise, depending on the initial conditions. In the catastrophic regime however, under particular choices of the initial conditions, the two rotational directions may coexist, with a portion of the agents going clockwise and the rest counterclockwise. This left and right rotational coexistence is not present in the $\mathrm{H}$-stable regime, where agents must keep a fixed distance from each other; in the catastrophic regime, on the other hand, it persists over very large simulation times and can be considered one of its hallmarks.

It is also important to note that the equations of motion giving rise to patterns of the type shown in Fig. 4 treat all individuals in the same way: there is no central commander and the motion of each agent depends only on its position relative to other members of the swarm. Our modeling is thus 


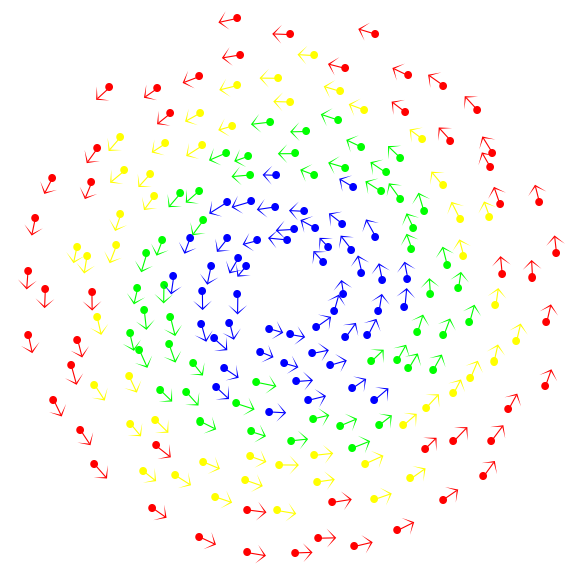

Fig. 6. Catastrophic vortex in the case of variable masses $m_{i}$, ranging continuously from 1.0 to 3.5. Only four colors are shown for these mass subgroups: from 1.0 to 1.7 blue; from 1.7 to 2.3 green; from 2.3 to 2.9 yellow; from 2.9 to 3.5 red. The red agents concentrated on the outer periphery, with higher masses, circulate with a larger radius. Those in blue are the lightest and describe smaller circles. Similar patterns arise in the case of uniform masses but variable self accelerations $\alpha_{i}$ or friction $\beta_{i}$. The interaction parameters are the same as in Fig. 5.

consistent with the fact that natural swarming occurs in a 'democratic' fashion with no leader emerging from the aggregate.

\section{Variations}

In this section we present more complex realizations of the model in Eqns.1-2. One interesting scenario is the usage of vehicle swarms of different masses $m_{i}$. Segregation is likely to occur. For the vortex scenario of region VII, for instance, all agents $i$ rotate at a constant speed $\left|v_{i}\right|^{2}=\alpha / \beta$ and experience a a mass dependent, centripetal force. This force must be balanced by the mass independent centrifugal term arising from the Morse potential. We obtain:

$$
\frac{m_{i} \alpha}{\beta r_{i}}=\left|\nabla_{i} U\left(\mathbf{x}_{i}\right)\right| .
$$

In this expression, the first term represents the centripetal force $m_{i}\left|v_{i}\right|^{2} / r_{i}$. From Eq. 5 , it is evident that the quantity $m_{i} / r_{i}$ must be constant: agents with larger masses segregate and tend to describe larger radii of motion, as seen 
in Fig. 6. Similar arguments can be applied to the case of same-mass agents when the $\alpha$ parameter is not chosen uniformly for all vehicles, but from a distribution $\alpha_{i}$ (or equivalently for $\beta$ ). In this case $\alpha_{i} / r_{i}$ is constant and similarly, agents with larger self-propulsion and faster speeds tend to describe larger radii of motion. For systems where the self-propulsion is programmed to be inversely proportional to the mass of the agent, vehicle segregation is no longer observed, in agreement with Eq. 5.

\section{Continuum limit}

Numerical simulations of the set of coupled, discrete equations of motion 1-3 are time consuming and generally for the systems shown here, one cannot go beyond a few thousand of interacting agents. In this section, we briefly outline the methodology for passing to the continuum limit, where discrete quantities are replaced by continuously varying fields that can offer insight for the collective behavior of large vehicle numbers. The continuum limit might also be useful to study systems for which the exact details of individual constituent motion are not necessary and a macroscopic, statistical picture is more pertinent. We coarse grain the equations of motion by following the work of Irving and Kirkwood [19] who first derived the macroscopic equations of hydrodynamics for a set of particles in a fluid. The density of the system is defined as:

$$
\rho(\mathbf{x})=\sum_{i} m_{i}\left\langle\delta\left(\mathbf{x}-\mathbf{x}_{i}\right) \mid f\right\rangle
$$

where brackets signify averaging over many configurations in the phase space defined by the position and momentum of the $i$ particles. The associated phase space probability distribution is denoted by $f$. Similarly, the velocity of the swarm $\mathbf{u}$ can be defined as:

$$
\rho(\mathbf{x}) \mathbf{u}(\mathbf{x})=\sum_{i} m_{i}\left\langle\mathbf{v}_{i} \delta\left(\mathbf{x}-\mathbf{x}_{i}\right) \mid f\right\rangle
$$

Using these definitions, the dynamics of individual agents, and ensemble averaging, one obtains the following conservation and transport laws [19, 12]:

$$
\begin{gathered}
\frac{\partial \rho}{\partial t}+\nabla \cdot(\mathbf{u} \rho)=0 \\
\frac{\partial \mathbf{u}}{\partial t}+(\mathbf{u} \cdot \nabla) \mathbf{u}=\alpha \mathbf{u}-\beta\left|\mathbf{u}^{2}\right| \mathbf{u}-\int \rho\left(x^{\prime}\right) \nabla U\left(\mathbf{x}-\mathbf{x}^{\prime}\right) d x^{\prime}
\end{gathered}
$$


where $U\left(\mathbf{x}-\mathbf{x}^{\prime}\right)$ is the two body Morse interaction. The derivation of these equations relies on the assumption that agents are not intrinsically correlated, so that the pair density $\rho^{(2)}\left(\mathbf{x}, \mathbf{x}+\mathbf{x}^{\prime}, t\right)$ can be written as the product of mass densities $\rho(\mathbf{x}, t) \rho\left(\mathbf{x}+\mathbf{x}^{\prime}, t\right)$. Furthermore it is assumed that velocity fluctuations and the energy flux into the system are negligible. In this case, local deviations of each agent velocity from the macroscopic group velocity are considered small. These assumptions are peculiar to the swarming system under consideration, where, for example, interaction terms are much stronger than statistical fluctuations. On a more fundamental note, the system described through Eqns. 1-3 is of a non-conserved type, whereas the standard Irving-Kirkwood derivation of the hydrodynamics equations assumes strictly Hamiltonian type forces. Coarse graining these systems depends on the classical Liouville equation for the time evolution of the position and momentum phase space density function $f$. In the present case, where there are dissipative terms, a strict usage of the Liouville equation is not possible, and a variant is needed [20].

The continuum model reproduces many features observed in the discrete case, and the phase diagram behavior anticipated in Fig. 2 is recovered. Counterclockwise and clockwise rotating structures are not observed anymore however, due to coarse graining. In particular, steady state solutions for flocking and vortex behavior may be found. In the first case, flocking will arise for $\mathbf{u}=\alpha / \beta \hat{z}$ where $\hat{z}$ is the flock direction and $\rho(r)=$ constant; vortex solutions correspond to $\mathbf{u}=\alpha / \beta(-\sin \theta, \cos \theta)$. In this case, the momentum equation gives an intrinsic formula for the density $\rho(r)$ as follows:

$$
\int_{0}^{\infty} \rho\left(\mathbf{r}^{\prime}\right) \nabla U\left(\mathbf{r}-\mathbf{r}^{\prime}\right) d \mathbf{r}^{\prime}=-\frac{\alpha}{\beta} \frac{\mathbf{r}}{|\mathbf{r}|^{2}} .
$$

This equation can be inverted by going to Fourier space and utilizing the convolution theorem. In the flocking case, a linear stability analysis around the uniform solution can be easily performed [20]. In the unstable regime, which corresponds to the catastrophic regime of potential parameters, perturbations will lead to the emergence of multiple, smaller rotating vortices, regularly spaced. We thus expect multiple spirals to arise in the discrete case as well, in the limit of large agent numbers - larger than the numbers used in the discrete systems analyzed here. A linear stability analysis can be performed in the case of vortex solutions as well, although the analysis is more complex. More details will be presented in Ref. [20].

\section{Further work and Conclusions}

Many issues still need to be addressed, such as determining typical swarm scaling as a function of $N$, both in the stable and catastrophic regimes, as well as the time required for pattern formation and whether these times can 
be cleverly expedited. In addition, when the interaction parameters are chosen to lie between the $\mathrm{H}$-stable to catastrophic transition the time for pattern formation increases and breathing phenomena occur within the emerging structures. It would be interesting to characterize these breathing modes. Furthermore, this study is of a deterministic type, but actual implementations of this model would involve the presence of stochastic terms, due to inevitable random sources of noise. Another issue of relevance is understanding the robustness of this multi-vehicular ensemble, both in the stable and catastrophic regimes, as well as its response to individual failure or in the presence of unwanted fields. Delays in communication between agents should be considered as well.

The goal of this work was to present various phases of aggregation for agents interacting through a tunable soft-core potential. Under certain conditions catastrophic behavior arises and in the limit of infinite constituents, the system collapses upon itself. It is easy to avoid such behavior: the insertion of a short-range infinite hard-core repulsion will make any potential stable. Collapsing behavior however, in spite of its name, might be quite useful in designing robot interactions: programming a stable to unstable crossover might lead the robots to change from a dispersive, searching mission to convergence at a specific site.

\section{Acknowledgments}

The authors wish to thank Herbert Levine, Daniel Marthaler and Tom Chou for useful discussions. We acknowledge support from ARO, ONR and NSF through grants W911NF-05-1-0112, N000140410054, and DMS-0306167.

\section{References}

1. N E Leonard, E Fiorelli (2001) Proceedings of the $40^{\text {th }}$ IEEE International Conference on Decision and Control 2968-2973 IEEE, Orlando

2. J Desai, V Kumar, J Ostrowski (1998) Proceedings of the IEEE International Conference on Robots and Automation 2864-2869

3. D Fox, W Burgard, H Kruppa and S Thrun (2000) Autonomous Robots 8:3

4. E W Justh, P S Krishnaprasad (2003) Proceedings of the $42^{\text {nd }}$ IEEE International Conference on Desicion and Control 3609-3614

5. S Camazine et al (2003) Self organization in biological systems Princeton Univ. Press, Princeton

6. J K Parrish, L Edelstein-Keshet (1999) Science 284:99-100

7. E Bonabeau, M Dorigo, G Theraulaz (1999) Swarm intelligence: from natural to artificial systems Oxford Univ Press, Oxford

8. M R D'Orsogna, Y L Chuang, A L Bertozzi, L S Chayes (2005) http://arxiv.org/abs/cond-mat/0509502

9. K Huang, (1987) Statistical Mechanics Wiley, New York 
10. D Ruelle (1969) Statistical Mechanics, Rigorous results, W A Benjamin Inc, New York

11. A. Procacci, Cluster expansion methods in rigorous statistical mechanics (www.mat.ufmg.br/aldo/papers/book.pdf).

12. H Levine, W J Rappel, I Cohen (2000) Phys Rev E 63:017101

13. R Hilborn (2001) Chaos and Nonlinear Dynamics Oxford Univ Press, Oxford

14. F Schweitzer, W Ebeling, B Tilch (2001) Phys Rev E 64:021110

15. B Q Nguyen et al (2005) Proc American Control Conference 1084-1089

16. V Gazi, K Passino (2002) Proceedings of the $41^{\text {st }}$ IEEE International Conference on Decision and Control 2842-2847 IEEE, Las Vegas.

17. V Gazi, K Passino (2003) IEEE Transactions on Automatic Control 48:692-697

18. G H Golub, J M Ortega (1992) Scientific Computing and Differential Equations: An Introduction to Numerical Methods Academic Press, New York

19. J H Irving, J G Kirkwood (1950) J Chem Phys 6:817-829

20. Y L Chuang in preparation 